\title{
Pathology for toxicologists?
}

Essentials of Pathology for Toxicologists edited by Paul Grasso, Taylor \& Francis, 2002. $£ 24.99$ (paperback) (166 pages) ISBN 0415257956

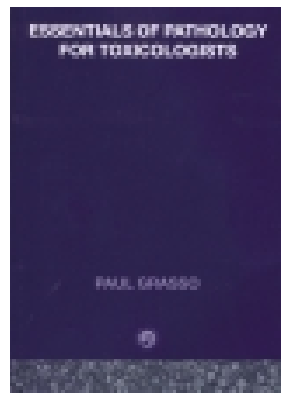

Essentials of Pathology for Toxicologists, edited and largely written by Paul Grasso, aims to makethebasic principles of pathology more understandablefor toxicologists. Although illustrated well with hand-drawings and computer illustrations and fortified with a rather useful glossary, the content of the book is very basic in its scientific breadth and thus would suit the needs of undergraduate students of biology and chemistry and associated fields as a basic demonstration of what pathology is about. However, the basic curricula in toxicology offered in North America and Europe to students encompass much more detailed knowledge of physiological and morphological properties of the organisms in question (including humans) than what is covered in this book. Furthermore, as an ideal help totoxicologists interested in pathology, in addition tothe drawings offered, photographs demonstrating themost typical type of lesions (e.g. necrosis, apoptosis, peroxisome proliferation, hyal ine droplets, fi brosis and amyloid, among others) would beessential. Indeed, the step from drawings to the picture seen under a microscope is a gigantic onefor someone unaccustomed totissue sections. Pathology for toxicologists would and should also involvetechniques of toxicological pathology employed by most veterinary and human pathologists in forensics and the drug and chemical industry. The detailed description of such techniques (e.g. in situ hybridization, immunohistochemistry and lasermicrodissection) or, at a minimum, an indication of whereto find theappropriate information is a must for toxicologists but is unfortunately missing in this book.
Somewhat disappointing is thechapter on carcinogenesis. This chapter lacks essential nomenclature. F or example, the type of cell (e.g. epithelial, mesenchymal or endothelial) from which the tumour has arisen, details on TNM (tumour sizeand spread, lymph-node involvement, absence/suspected/presence of metastases) classification schemes and how these were devised, in addition to a rough description of the basic mechanisms of tumour initiation, promotion, progression and metastasis areall omitted. Although a table is presented describing the features of benign and malignant tumours, the labels of the individual tablecolumns have been interchanged such that the student cannot derive which of the characteristics belong to the malignant versus the benign tumour.

A most important part of pathology for toxicologists involves the real ization that surrogate animals used for oncology and toxicology research might be predisposed for the devel opment of certain tumours [1] (e.g. B6C3F 1 micearesusceptible to liver tumours and malerats are susceptibleto chronic progressive nephropathy and kidney tumours as a result of the presence of $\alpha 2 \mathrm{u}$-globulin) and that thesetypes of tumours and their aetiology might not be readily extrapolated to humans. Similarly, a morethorough and detailed presentation of viral-induced tumours would have been of great benefit, particularly in conjunction with a brief description of how these viruses can be detected in cytological and histological preparations.

Although also presented in a very basic and brief fashion, the chapter on immunology appears useful and helpful and provides for a solid understanding of the main principles and players. This unfortunately cannot besaid for the chapters on clinical chemistry and haematology. Although the chapter on clinical chemistry lacks thorough examples that allow understanding of theramifications of the parameters determined, the chapter on haematology is severely deficient in illustrations.

The best part of this book is certainly the glossary; however, in all honesty, a more profound and broader gl ossary can befound on the internet [e.g. Toxnet maintained by the National Library of Medicine(http://toxnet.nlm.nih.gov)]. Therefore, the conclusion is that this book, although intended for toxicol ogists, is a limited and superficial overview for the interested layman or the beginner student in biological and chemical sciences.

\section{Daniel Dietrich}

Environmental Toxicology, University of Konstanz, Konstanz, Germany. e-mail: Daniel.Dietrich@uni-konstanz.de

\footnotetext{
Reference

1 Swenberg, J .A. et al. (1992) Species specific mechanisms of carcinogenesis. In Mechanisms of Carcinogenesis in Risk I dentification (Vainio, H. et al., eds), pp. 470-500, Lyon International Agency for Research on Cancer
} 